\title{
Proposal of Chlamydia pecorum sp. nov. for Chlamydia Strains Derived from Ruminants
}

\author{
HIDETO FUKUSHI AND KATSUYA HIRAI* \\ Department of Veterinary Microbiology, Faculty of Agriculture, \\ Gifu University, Yanagido 1-1, Gifu 501-11, Japan
}

\begin{abstract}
Chlamydia pecorum sp. nov. is proposed as the fourth species of the genus Chlamydia on the basis of the results of a genetic analysis of Chlamydia strains that were isolated from cattle and sheep which had various diseases, including sporadic encephalitis, infectious polyarthritis, pneumonia, and diarrhea. The levels of DNA-DNA homology between $C$. pecorum and strains of $C$. psittaci, Chlamydia pneumoniae, and Chlamydia trachomatis were less than $10 \%$. Several DNA probes were used to identify $C$. pecorum. The $C$. pecorum strains were distinguished from $C$. psittaci strains by the results of immunological assays, including an immunofluorescence antibody assay performed with monoclonal antibodies and an immunoblot analysis of the immunological specificity of the major outer membrane protein. Species identification was based on results obtained from DNA analyses and serology. The type strain of $C$. pecorum is strain ATCC VR628.
\end{abstract}

The members of the genus Chlamydia are obligately intracellular gram-negative bacteria that have a unique developmental cycle. The following three species have been recognized previously in this genus: Chlamydia trachomatis, Chlamydia psittaci, and Chlamydia pneumoniae $(8,13) . C$. trachomatis and $C$. psittaci were the first two species assigned to the genus Chlamydia on the basis of differences in inclusion morphology, glycogen content, susceptibility to sulfadiazine, and natural hosts $(7,15)$. C. pneumoniae has an inclusion morphology similar to that of $C$. psittaci. However, $C$. pneumoniae is separated from the other two species on the basis of ultrastructural differences in the elementary bodies, DNA homology values, and serological reactions (8). $C$. trachomatis and C. pneumoniae are human pathogens, while $C$. psittaci is an animal pathogen. $C$. psittaci strains have been isolated from more than 130 species of birds and from many species of mammals, including cattle, sheep, goats, cats, muskrats, guinea pigs, and koala bears (21). C. psittaci causes a wide variety of diseases in animals, including encephalomyelitis, pneumonia, polyarthritis, enteritis, conjunctivitis, mastitis, abortion, and other diseases (21).

The species $C$. psittaci encompasses a heterogeneous group of organisms that differ genetically, serologically, and in host tropism. Many efforts have been made to clarify relationships among $C$. psittaci strains. Generally, $C$. psittaci can be divided into avian and mammalian groups. The mammalian group of $C$. psittaci also exhibits heterogeneity. A DNA-DNA homology analysis of isolates obtained from cattle and sheep that had pneumonia, polyarthritis, encephalomyelitis, and diarrhea showed that these isolates exhibited less than $15 \%$ homology with other members of $C$. psittaci and with $C$. trachomatis and $C$. pneumoniae strains (5; unpublished data). One exception was ovine abortion strain Ov/B577, which exhibited more than 50\% DNA sequence similarity with avian strains. A DNA restriction endonuclease analysis revealed diverse patterns in $C$. psittaci strains that were derived from birds and mammals. Differences between ovine polyarthritis strains and ovine abortion strains have also been observed $(5,11,22)$. Several reports have indicated that different immunological and

\footnotetext{
* Corresponding author.
}

biological types are represented in $C$. psittaci strains isolated from cattle and sheep $(2,3,16-18,20)$.

On the basis of the data described above, we propose that strains which are isolated from cattle and sheep and cause pneumonia, polyarthritis, encephalomyelitis, and diarrhea should be assigned to a new species, Chlamydia pecorum.

Justification for a new species. The first $C$. pecorum strain that was isolated, strain Bo/E58 ${ }^{\mathrm{T}}$ (= ATCC VR628 $\left.{ }^{\mathrm{T}}\right)(\mathrm{T}=$ type strain), was isolated in 1953 from the brain of a calf that had sporadic bovine encephalomyelitis, and the organism was identified as a member of the genus Chlamydia $(12,23)$. Subsequently, many strains have been isolated from cattle and sheep that have pneumonia, encephalomyelitis, and polyarthritis and from feces of healthy animals $(14,19)$. $C$. pecorum strains were originally classified as $C$. psittaci strains on the basis of inclusion morphology (inclusions are dense and oval and contain no glycogen) and resistance to sulfadiazine (7). The ultrastructural morphology of $C$. pecorum elementary bodies is typical of the morphology of $C$. psittaci and C. trachomatis elementary bodies (Fig. 1).

The justification for separating $C$. pecorum strains from $C$. psittaci strains and establishing a new species for the $C$. pecorum strains is based on the results of DNA and immunological analyses.

A genetic analysis showed that $C$. pecorum strains exhibited $20 \%$ or less DNA relatedness to strains of the other three Chlamydia species, while the levels homology were $88 \%$ or greater within the species (Table 1) (5). The guanineplus-cytosine contents of the DNAs were 39.3 mol\% for $C$. pecorum, $39.6 \mathrm{~mol} \%$ for C. psittaci, $39.8 \mathrm{~mol} \%$ for $C$. trachomatis, and $40.3 \mathrm{~mol} \%$ for C. pneumoniae (9) (Table 2). The DNA restriction endonuclease digestion patterns of $C$. pecorum isolates were identical and were readily distinguishable from those of $C$. trachomatis, $C$. pneumoniae, and $C$. psittaci strains (5). C. pecorum could also be differentiated from the other chlamydiae by the results of Southern blotting when either whole chromosomal DNA or cloned $C$. pecorum restriction DNA fragments were used as the probes (manuscript in preparation). C. pecorum isolates could also be distinguished by the results of an immunoblot analysis in which hyperimmune sera were used or by the results of an immunofluorescence test in which monoclonal antibodies were used $(4,6)$. At least three serovars were identified in 


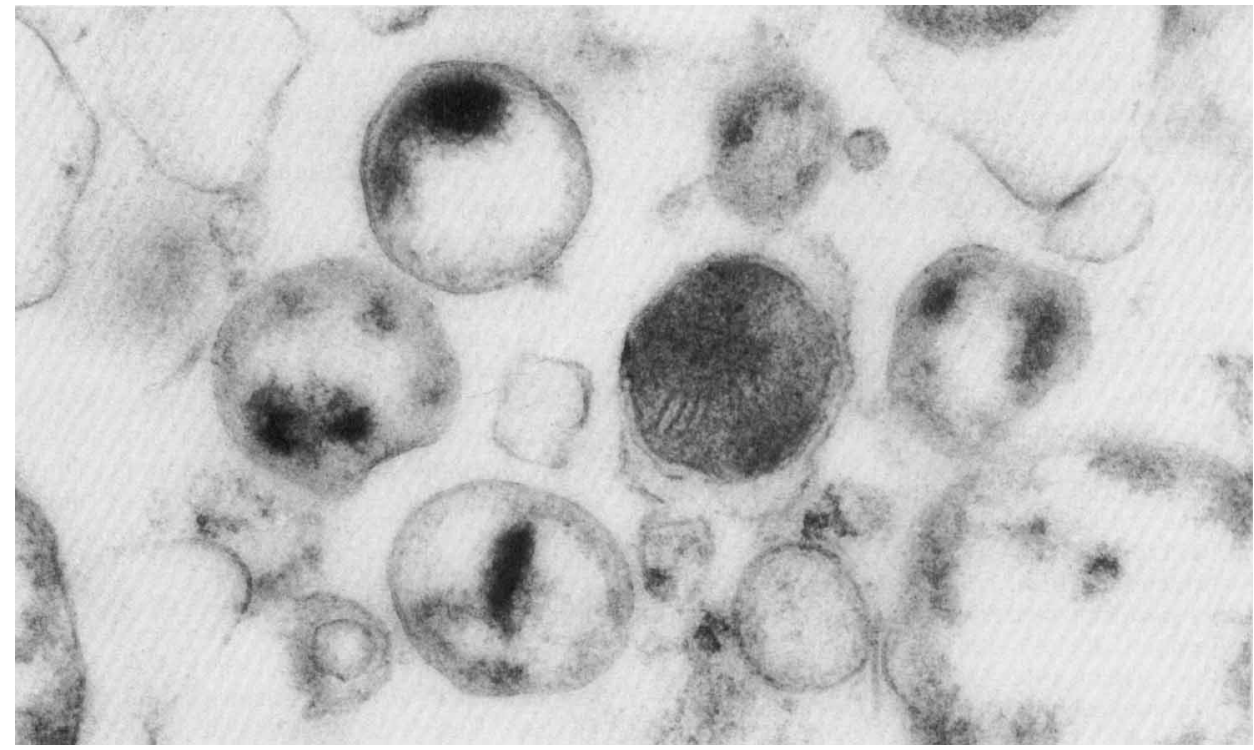

FIG. 1. Electron micrograph of a section of purified elementary bodies of strain Bo/E58 $8^{\mathrm{T}}(=\mathrm{ATCC}$ VR628 $)$.

this species by a immunoblot analysis of the major outer membrane protein (4).

Description of Chlamydia pecorum sp. nov. Chlamydia pecorum (pe.co'rum. M. L. gen. pl. pecorum, flocks of sheep or herds of cattle). The description below is based on the results obtained with the following strains: strains Bo/ $\mathrm{E}^{\mathrm{T}}{ }^{\mathrm{T}}$ (= ATCC VR628 ${ }^{\mathrm{T}}$ ) and Bo/Shizuoka, which were isolated from cattle that had encephalomyelitis; strain $\mathrm{Bo} /$ Maeda, which was isolated from cattle that had pneumonia; strain Bo/Yokohama, which was isolated from cattle that had diarrhea; and strain Ov/IPA (= ATCC VR629), which was isolated from sheep that had polyarthritis. C. pecorum has many phenotypic characteristics that are similar to those described previously for $C$. psittaci, including the developmental cycle, the ultrastructural morphology of the elementary body and the reticulate body, the inclusion morphology, the absence of glycogen in inclusions, and sulfadiazine resistance. It contains the Chlamydia genus-specific lipopolysaccharide antigen, as demonstrated by the complement fixation test. The guanine-plus-cytosine content of the DNA is $39.3 \mathrm{~mol} \%$. C. pecorum can be isolated by inoculating chicken embryo yolk sacs and cell cultures containing Madin-Derby bovine kidney and HeLa cells. Cell culture growth is enhanced by pretreating cells with DEAE-

TABLE 1. Levels of DNA-DNA homology for C. pecorum, C. psittaci, C. trachomatis, and C. pneumoniae ${ }^{a}$

\begin{tabular}{lcccc}
\hline \multirow{2}{*}{ Species } & \multicolumn{4}{c}{$\%$ Homology with: } \\
\cline { 2 - 5 } & C. pecorum & C. psittaci & C. trachomatis & C. pneumoniae \\
\hline C. pecorum & $88-100$ & $1-19$ & $1-10$ & 10 \\
C. psittaci & & $14-95$ & $1-33$ & $1-8$ \\
C. trachomatis & & $20-92^{h}$ & $1-7$ \\
C. pneumoniae & & & $94-96$ \\
\hline
\end{tabular}

${ }^{a}$ DNA-DNA homology values were taken from references 1 and 5 and our unpublished data.

$b$ The levels of DNA-DNA homology were more than $92 \%$ between strains belonging to biovars trachoma and lymphogranuloma and $20 \%$ between a mouse biovar strain and strains belonging to other biovars. dextran before inoculation and incubating infected cells with media containing cycloheximide $(20)$. C. pecorum has been isolated from brains, lungs, joints, embryonic kidneys, feces, and other parts of cattle and sheep. The diseases caused by $C$. pecorum include encephalomyelitis, polyarthritis, pneumonia, and enteritis. Three serovars are distinguished by the results of immunoblot analyses of the major outer membrane protein. The type strain is strain Bo/E58 (= ATCC VR628), which is the first strain of this species that was isolated. Strain Bo/E58 ${ }^{\mathrm{T}}$ can be propagated in MadinDerby bovine kidney cells and has characteristics typical of C. pecorum.

We thank C.-C. Kuo, L. A. Campbell, M. P. Melgosa, and T. MacAdoo for their helpful comments and suggestions for revising the manuscript.

A part of this work was supported by Grants-in-Aid for Scientific Research from the Ministry of Education, Science and Culture of Japan.

TABLE 2. Guanine-plus-cytosine contents of C. pecorum, C. psittaci, C. trachomatis, and C. pneumoniae

\begin{tabular}{lcccc}
\hline \multirow{2}{*}{ Source of data } & \multicolumn{4}{c}{ Guanine-plus-cytosine content (mol\%) of: } \\
\cline { 2 - 5 } & C. pecorum & C. psittaci & C. trachomatis & C. pneumoniae \\
\hline This study & $39.3^{a, b}$ & $39.6^{a, c}$ & $39.8^{a, d}$ & $40.3^{a, e}$ \\
Previous studies & $\mathrm{NA}^{g}$ & $39-43$ & $42-45$ & 40 \\
\hline
\end{tabular}

${ }^{a}$ Values were estimated by using the P1 nucleases high-performance liquid chromatography method (9); 2 to $5 \mu \mathrm{g}$ of DNA was denatured by heat and was digested with $\mathrm{P} 1$ nuclease. The resulting nucleotides were then dephosphorylated with alkaline phosphatase and analyzed by using a type TSK-GEL ODS-80TM column.

${ }^{b}$ Average for five strains, strains Bo/E58 ${ }^{\mathrm{T}}$ (= ATCC VR628 ${ }^{\mathrm{T}}$ ), Bo/Yokohama, Bo/Maeda, Bo/Shizuoka, and Ov/IPA (= ATCC VR629).

${ }^{c}$ Average for 18 strains, including strain Prk/6BC (= ATCC VR125), the type strain of $C$. psittaci.

${ }^{d}$ Average for three strains, including strain L2/434/Bu (= ATCC VR121), the type strain of $C$. trachomatis.

$e$ Value for the type strain, C. pneumoniae TW183.

${ }^{f}$ Data from references 1 and 10 .

$g$ NA, not available. 


\section{REFERENCES}

1. Cox, R. L., C.-C. Kuo, J. T. Grayston, and L. A. Campbell. 1988. Deoxyribonucleic acid relatedness of Chlamydia sp. strain TWAR to Chlamydia trachomatis and Chlamydia psittaci. Int. J. Syst. Bacteriol. 38:265-268.

2. DeLong, W. J., and W. E. Magee. 1986. Distinguishing between ovine abortion and ovine arthritis Chlamydia psittaci isolates with specific monoclonal antibodies. Am. J. Vet. Res. 47:1520 1523.

3. Eb, F., and J. Orfila. 1982. Serotyping of Chlamydia psittaci by the microimmunofluorescence test: isolates of ovine origin. Infect. Immun. 37:1289-1291.

4. Fukushi, H., and K. Hirai. 1988. Immunochemical diversity of the major outer membrane protein of avian and mammalian Chlamydia psittaci. J. Clin. Microbiol. 26:675-680.

5. Fukushi, H., and K. Hirai. 1989. Genetic diversity of avian and mammalian Chlamydia psittaci strains and relation to host origin. J. Bacteriol. 171:2850-2855.

6. Fukushi, H., K. Nojiri, and K. Hirai. 1987. Monoclonal antibody typing of Chlamydia psittaci derived from avian and mammalian species. J. Clin. Microbiol. 25:1978-1981.

7. Gordon, F. B., and A. L. Quan. 1965. Occurrence of glycogen in inclusions of the psittacosis-lymphogranuloma venereum-trachoma agents. J. Infect. Dis. 115:186-196.

8. Grayston, J. T., C.-C. Kuo, L. A. Campbell, and S.-P. Wang. 1989. Chlamydia pneumoniae sp. nov. for Chlamydia sp. strain TWAR. Int. J. Syst. Bacteriol. 39:88-90.

9. Kaneko, T., K. Katoh, M. Fujimoto, M. Kumagai, J. Tamàoka, and Y. Katayama-Fujimura. 1986. Determination of the nucleotide composition of a deoxyribonucleic acid by high-performance liquid chromatography of its enzymatic hydrolysate: a review. J. Microbiol. Methods 4:229-240.

10. Kingsbury, D. T., and E. Weiss. 1968. Lack of deoxyribonucleic acid homology between species of the genus Chlamydia. J. Bacteriol. 96:1421-1423.

11. McClenaghan, M., A. J. Herring, and I. D. Aitken. 1984. Comparison of $C$. psittaci isolates by DNA restriction endonuclease analysis. Infect. Immun. 45:384-389.
12. McNutt, S. H., and E. F. Waller. 1940. Sporadic bovine encephalomyelitis. Corriell Vet. 30:437-448.

13. Moulder, J. W., T. P. Hatch, C.-C. Kuo, J. Schachter, and J. Storz. 1984. Chlamydia Jones, Rake and Stearns 1945, 55, p. 729-735. In N. R. Krieg and J. G. Holt (ed.), Bergey's manual of systematic bacteriology, vol. 1. The Williams \& Wilkins Co., Baltimore.

14. Omori, T., S. Ishi, and M. Matsumoto. 1960. Miyagawanellosis of cattle in Japan. Am. J. Vet. Res. 21:564-573.

15. Page, L. A. 1968. Proposal for the recognition of two species in the genus Chlamydia Jone, Rake and Stearns, 1945. Int. J. Syst. Bacteriol. 18:51-66.

16. Perez-Martinez, J. A., and J. Storz. 1985. Antigenic diversity of Chlamydia psittaci of mammalian origin determined by microimmunofluorescence. Infect. Immun. 50:905-910.

17. Schachter, J., J. Banks, N. Sugg, M. Sung, J. Storz, and K. F. Meyer. 1974. Serotyping of Chlamydia. I. Isolates of ovine origin. Infect. Immun. 9:92-94.

18. Schachter, J., J. Banks, N. Sugg, M. Sung, J. Storz, and K. F. Meyer. 1975. Serotyping of Chlamydia. II. Isolates of bovine origin. Infect. Immun. 11:904-907.

19. Shewen, P. E. 1980. Chlamydial infection in animals. A review. Can. Vet. J. 21:2-11.

20. Spears, P., and J. Storz. 1979. Biotyping of Chlamydia psittaci based on inclusion morphology and response to diethylaminoethyl-dextran and cycloheximide. Infect. Immun. 24:224-232.

21. Storz, J. 1988. Overview of animal diseases induced by chlamydial infections. p. 167-192. In A. L. Barron (ed.), Microbiology of Chlamydia. CRC Press, Inc., Boca Raton, Fla.

22. Timms, P., F. W. Eaves, A. A. Girjes, and M. F. Lavin. 1988. Comparison of Chlamydia psittaci isolates by restriction endonuclease and DNA probe analyses. Infect. Immun. 56:287-290.

23. Wenner, H. A., G. S. Harshfield, T. W. Chang, and R. W. Menges. 1953. Sporadic bovine encephalomyelitis. II. Studies on the etiology of the disease, isolation of nine strains of an infectious agent from naturally infected cattle. Am. J. Hyg. 57:15-29. 